\title{
From system ecology to urban morphology: towards a theory of urban form resilience
}

\author{
Alessandra Feliciotti ${ }^{1, *}$, Ombretta Romice ${ }^{2}$ and Sergio Porta $^{3}$ \\ ${ }^{1}$ Research associate at Urban Design Studies Unit (UDSU), Department of Architecture, Faculty of \\ Engineering, University of Strathclyde. \\ 2 Senior Lecturer at Urban Design Studies Unit (UDSU), Department of Architecture, Faculty of \\ Engineering, University of Strathclyde. \\ ${ }^{3}$ Professor at Urban Design Studies Unit (UDSU), Department of Architecture, Faculty of \\ Engineering, University of Strathclyde
}

E-Mails: a.feliciotti@strath.ac.uk; ombretta.r.romice@strath.ac.uk; sergio.porta@strath.ac.uk

* Author to whom correspondence should be addressed; Tel.: +44-(0)141 548 3171;

\begin{abstract}
As cities grow in scale and complexity, the extent to which their urban forms will be able resist, adapt to or co-evolve under unpredictable circumstances and fulfil needs different from those they were originally designed for, may be crucial for the very survival of cities. In this context, the concept of resilience, originated in ecology as a way to deal with change and uncertainty in ecological systems, particularly in its 'evolutionary' interpretation, has gained salience in relation to urban systems where, not unlike in other kinds of complex adaptive systems, change can both be triggered by external idiosyncratic shocks and emerge gradually from internal processes of self-organisation, and is now considered as pivotal for the design and management of the built environment. Whilst several authors have tried to build a bridge between resilience thinking and urban design, the role of the morphological structure of cities in enabling or constraining resilient responses has never been addressed systematically and, indeed, evidence that the framework of evolutionary resilience can be extended to the urban form is hardly systematic. To overcome this gap, this article seeks to evidence the link between urban form and resilience theory. This is done by building a parallel between concepts, models and organisational principles developed in system ecology to explain dynamics of change in ecosystems (i.e. Adaptive Cycles, Panarchy), to analogous models developed independently in the discipline of urban morphology to describe dynamics
\end{abstract}


of change in urban form (i.e. Burgage Cycle, Territorial Development Cycle, Urban Form Compositional Hierarchy). On this basis, a new theoretical model of urban form change grounded on an understanding of urban form as complex system, is formalised, substantiating the application of evolutionary resilience urban form.

Keywords: resilience; urban form; urban morphology; system ecology.

\section{Resilience and urban form: where are we at?}

In coming years and decades, the form of our cities will be called to meet new needs and requirements by preserving, adapting or transforming their structure in response to pressures for change - internal or external, gradual or sudden, predictable or unpredictable. On this capacity will depend the ability of cities to survive and even thrive. This awareness brought the concept of resilience, particularly in its evolutionary connotation, to the forefront of the debate in urban design, where it is used to better understand the process of continuous change and adaptation characterising complex urban systems in conditions of uncertainty and address their design and management (Pickett et al. 2013). Several authors tried to build a common ground between resilience thinking and urban design, also expressing an interest in understanding how urban form in particular can be approached from a resilience perspective (Marcus and Colding, 2014; Anderies, 2014) but whilst this is encouraging, few explicitly investigate the contribution of the micro-scale spatial structure of cities in enabling or constraining resilient responses to socio-economic fluctuations, and make virtually no reference to fundamental morphological components, as plots, streets and blocks, which are, in turn, central in urban design. Crucially, to be meaningful for urban designers, resilience cannot leave urban form aside.

Furthermore, research shows that geometrical and configurational properties of urban form components (i.e. granularity of plots, the size of blocks, connectivity of streets) influence the extent to which places can preserve unique identities, adapt to new needs and innovate (Brand, 1995, Tachieva, 2010, Dovey, 2016), all aspects that are central to the resilience discourse. And yet, there is skepticism towards the idea that, beyond metaphors, urban form can truly be addressed from a resilience perspective, largely because urban form is not commonly perceived as a complex adaptive system itself. Indeed, central models of resilience theory are hardly ever directly applied to urban form.

Urban form is very much related with resilience because it is structured and behaves not unlike other kinds of complex adaptive systems. A comparison between system ecology, the discipline that formulated many core concepts of resilience theory, and urban morphology the discipline that studies the urban form of cities help us show how.

\section{System Ecology and Urban morphology: not so different after all}

At first sight, system ecology and urban morphology could not appear more different: one is a branch of natural sciences concerned with the study of ecological communities and their habitats, the other is a field of enquiry that studies shape, layout and composition of built environment and open spaces. And 
yet,they have several points of contact. First, both urban morphology and system ecology - particularly its specialist branch, landscape ecology - share a common interest in the physical space: the first being the abiotic spatial structure of cities and the second being the biotic dimension of landscapes. Then, both can be considered as systemic disciplines, as they look at their respective object of investigation in relational terms, giving predominance to the study of pattern than to the study of matter (Capra and Luisi, 2014): system ecologists focus on the interactions between species and their habitats, whilst urban morphologists study the constant feedback between people and built environment. Finally, both have a strong focus on change through time: system ecology studies the patterns of change in structure and behavior of ecosystems and urban morphology addresses the formation and transformation of urban forms in different contexts.

But there is more to it. Crucially, core concepts and hypotheses formulated by system ecologists to describe the structure and dynamics of change in ecological systems are almost exactly mirrored in urban morphology, with regards to the multi-scalar organisation and processes of genesis and transformation of morphological elements.

\section{Cycles of change: Adaptive Cycle and Urban Form Cycles}

According to system ecology, throughout their existence, ecosystems are never still but, broadly speaking, go through four different phases of a cyclical process known as "Adaptive Cycle", as a sort of "heuristic theory of change" (Gunderson and Holling, 2001): a phase of gradual growth (Exploitation), where dominant species take stage and available resources are colonized, a phase of consolidation of accumulated energy, capital and resources (Conservation), a sudden and abrupt moment of collapse triggered by an external or internal disturbance (Release) and, finally, a final phase where, exploiting the creative element inherent in the disruption, the system starts reorganizing for a new cycle. In doing so, the system can reform around the same sets of variables, in a repetition of the previous cycle, or, more rarely, it can shift to a new regime characterised by different processes and structures and thus a new trajectory (Figure 1).

Figure 1. Adaptive cycle in its four phases. Exploitation and Conservation, Release and Reorganisation, based on Holling and Gudnerson (2001). 


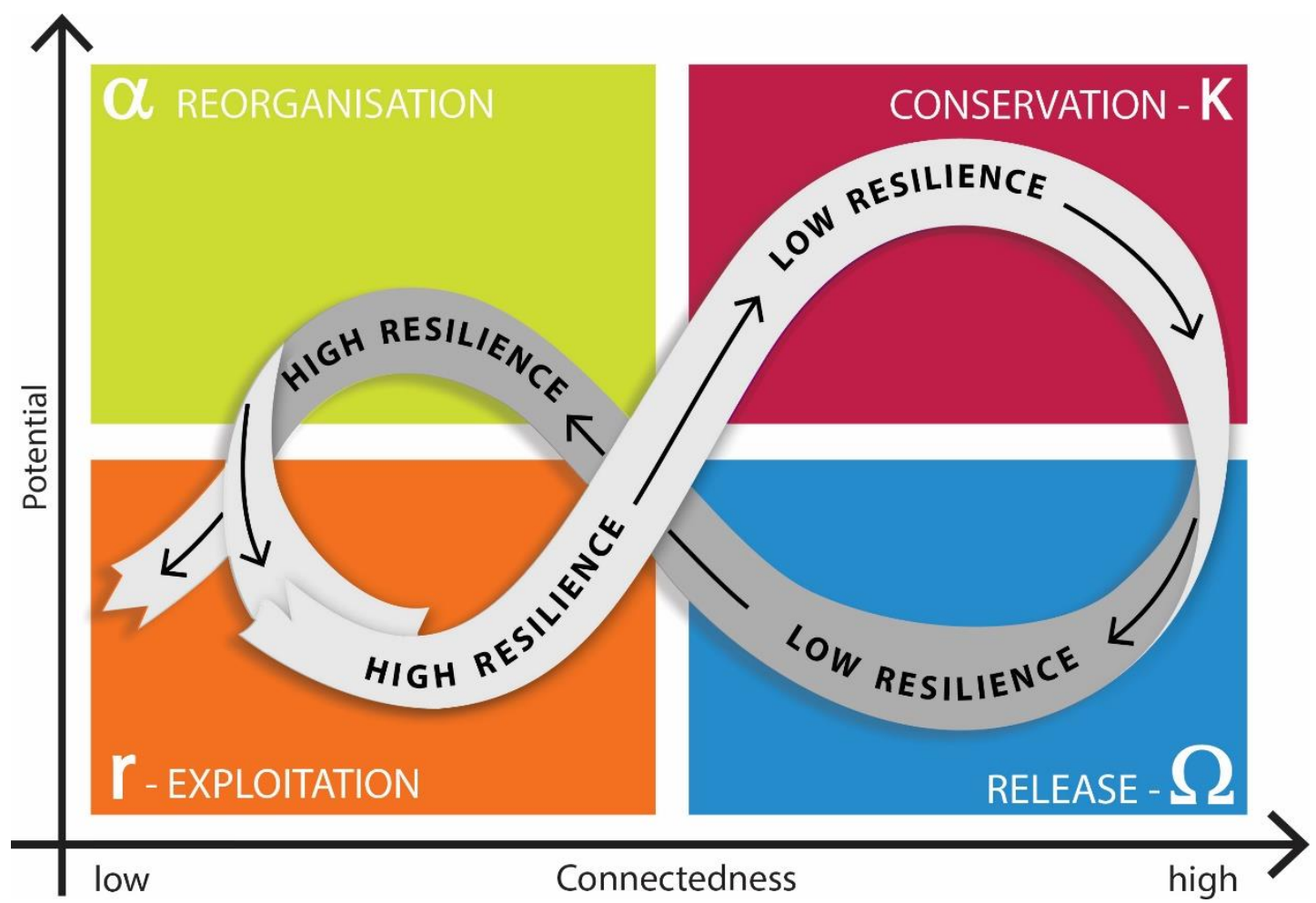

Source: Feliciotti A., 2018, Resilience and Urban Design: A systems approach to the study of resilience in urban form. $\mathrm{PhD}$ Thesis, Department of Architecture, University of Strathclyde.

Since its formulation, the Adaptive Cycle has been observed across a variety of complex systems other than ecosystems, including social, economic and urban systems, albeit never in urban form. However, according to urban morphologists, urban form also experience periods of creation, growth, collapse and reinstitution, which, beyond variability of contextual outcomes, also happens to follows a cycle (Kropf, 2001)

This process was first described at the scale of plots by geographer Conzen in 1960 in the British

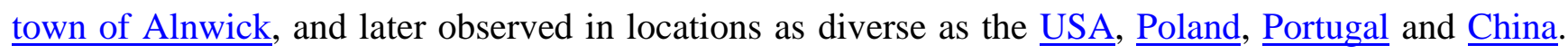
This is known as "Burgage Cycle" and is divided into four phases: an Institutive phase, corresponding to the establishment of the initial plot structure, a Repletive phase, where open space of each plot is progressively infilled, a Climax phase where, existing plot structure is maximally exploited to a point of saturation, a Recessive phase, characterised by a rapid drop in density and building coverage leading to a partial or total obliteration of the entire plot pattern and, finally a new institutive phase which can either adapt to pre-existing street system or profoundly altering the relationship previously existing between morphological components (Figure 2).

It is quite easy to see how the different periods of the Burgage cycle are, at all effects, a morphological equivalent of the cycle of exploitation, conservation, release and reorganisation described in the Adaptive cycle. Additionally, in both models, the transition from one phase to the next is configured as discrete responses to the wider context (i.e. changes in society, technology, climate etc.) and each cycle is influenced by past system configuration through path dependency, and as such it is unique. But this is just part of the story... 
Figure 2. Different phases of the Burgage Cycle by Conzen (1960), expressed as variation in building coverage over time.

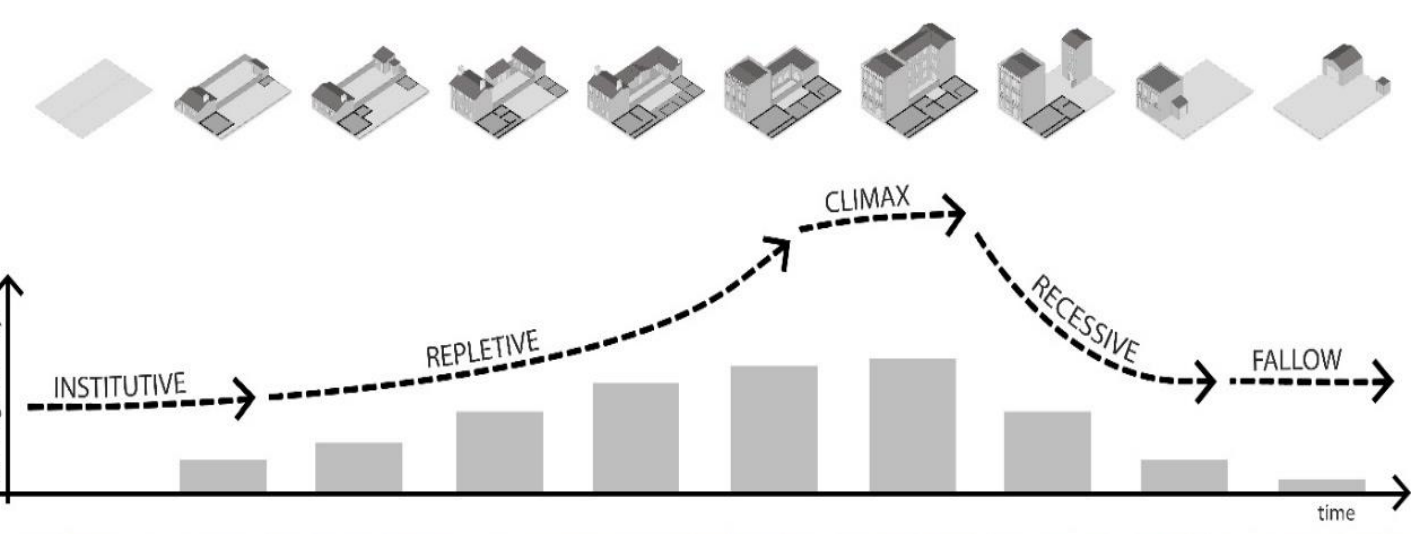

Source: Feliciotti A., 2018, Resilience and Urban Design: A systems approach to the study of resilience in urban form. $\mathrm{PhD}$ Thesis, Department of Architecture, University of Strathclyde.

\section{Dynamic structural hierarchy: Panarchy and Compositional Hierarchy.}

In ecosystems, or more generally, in all kind of complex systems, the succession of stages described in the Adaptive Cycle can be observed simultaneously at the different scales, from smallest structures (i.e. the leaves in a tree) to the very largest ones (i.e. the biosphere), although these happen at different speed and frequency, with small-scale structures having adaptive cycles unfolding over weeks or months, and larger structures undergoing century-long adaptive cycles. And whilst, at each scale, the unfolding of the cycle depends on a limited number of scale-specific variables and is therefore relatively compartmentalised, there is always constant feedback with levels above and below. Because large-scale elements have slower adaptive cycles, these exert a conservative force on lower and faster scales, providing a continuity to system and hampering low-level innovation to scale up, a mechanism called "remember". At the same time, under particular circumstances, lower scales have the power to trigger transformations from the bottom-up, overwhelming higher-level structures and propagating small-scale novelties throughout the system, a mechanism is called "revolt" (Gunderson and Holling, 2001). This complex organizational model linking scales in space in time is known as Panarchy (figure 3).

Figure 3. Panarchy applied to a forest ecosystem. The diagram shows a four-tiered hierarchy with relevant structures appearing at different scales of space and time. Elaboration of the author from Gunderson and Holling, (2001). 


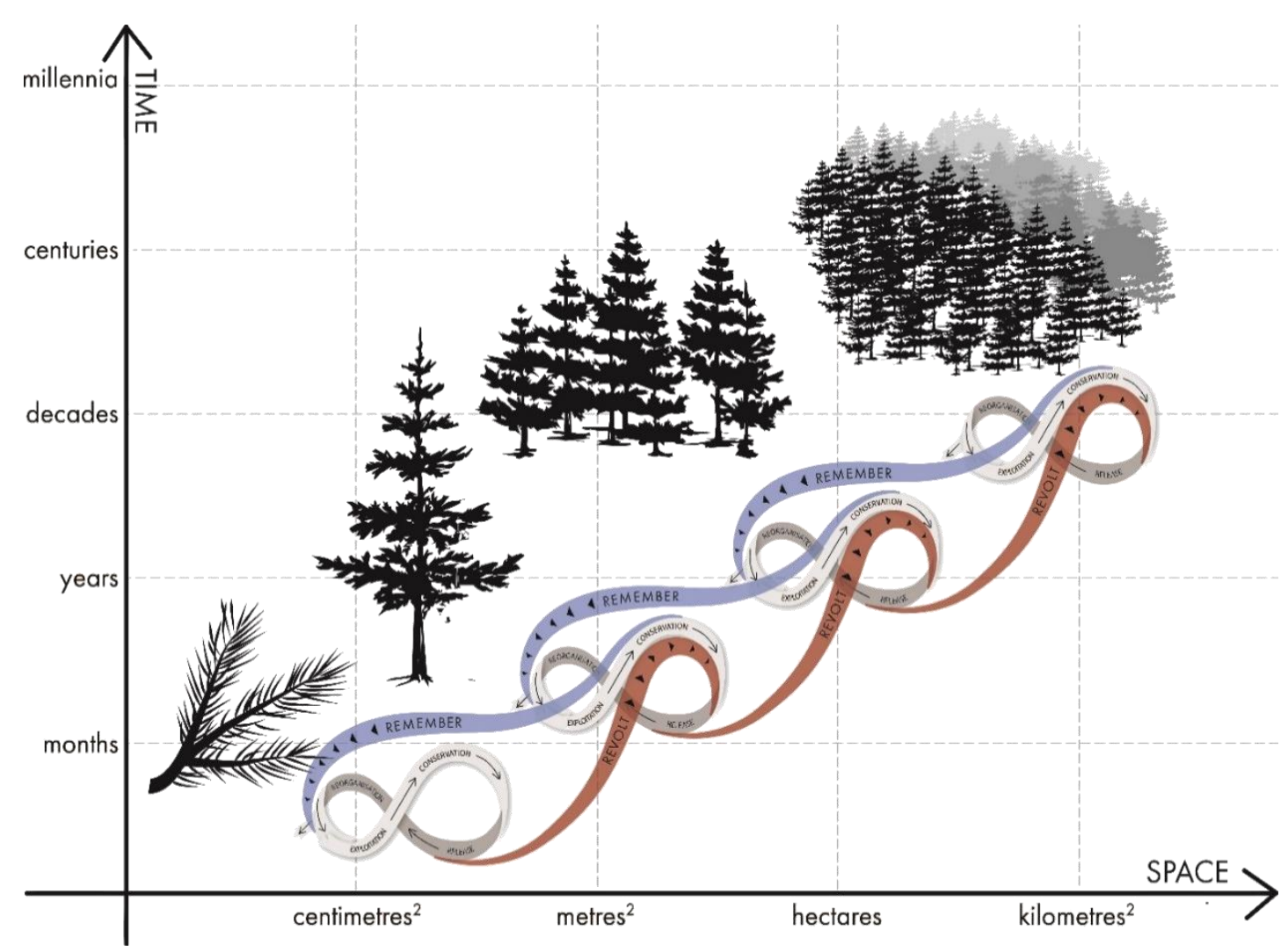

Feliciotti A., 2018, Resilience and Urban Design: A systems approach to the study of resilience in urban form. $\mathrm{PhD}$ Thesis, Department of Architecture, University of Strathclyde.

In Urban Morphology, there is no explicit model equivalent to the Panarchy. However, implicitly, all pieces are already there. Urban morphologists understand urban form as a "compositional hierarchy", an organisational structure where small-scale spatial elements combine to form larger-scale elements in a part-to-whole relationship. Buildings and their pertinent open area form plots, series of plots accessed from the same street form street edges, contiguous street edges form blocks, the space carved out from blocks forms streets, and so on, in a multi-level structure that is "infinitely extensible in logical terms" (Kropf 2014).

Whilst the compositional hierarchy is a predominantly spatial construct, at the same time, urban morphologists recognise that the various components occupying its levels have different lifespans and different attitudes to inertia/changeability. For example, the street system opposes considerable resistance to change once laid down, reflecting capital investment, whist plots are comparatively more changeable, through subdivision and amalgamations. Buildings are even more susceptible, as demolition and replacement are rather common (i.e. obsolescence). Their interior, in turn can be modified quite frequently through change of use and repartitioning.

This idea of duration of spatial elements has a lot to do with the Conzen's Burgage Cylce which, in fact, is not at all unique to plots but exists at different scales: Stewart Brand (1995) described it at the scale of buildings, whilst Italian morphologists Caniggia and Maffei (1979) observed it at the scale of entire urban settlements and called it "territorial development cycle". Crucially, these are but specific illustrations of more a more general process taking place at each and every scale of the urban form, albeit at different speeds. And, since the morphological components co-exist in same urban environment, these cycles unfold simultaneously. All of this adds a temporal dimension to the compositional hierarchy, 
closely recalling the nested structure suggested by the Panarchy (Gunderson and Holling, (2001), where smaller components with faster cycles nest within larger components within slower cycles. (Figure 4).

Figure 4. The Panarchy in urban form. The diagram shows a four-tiered hierarchy with relevant morphological structures appearing at different scales of space and time.

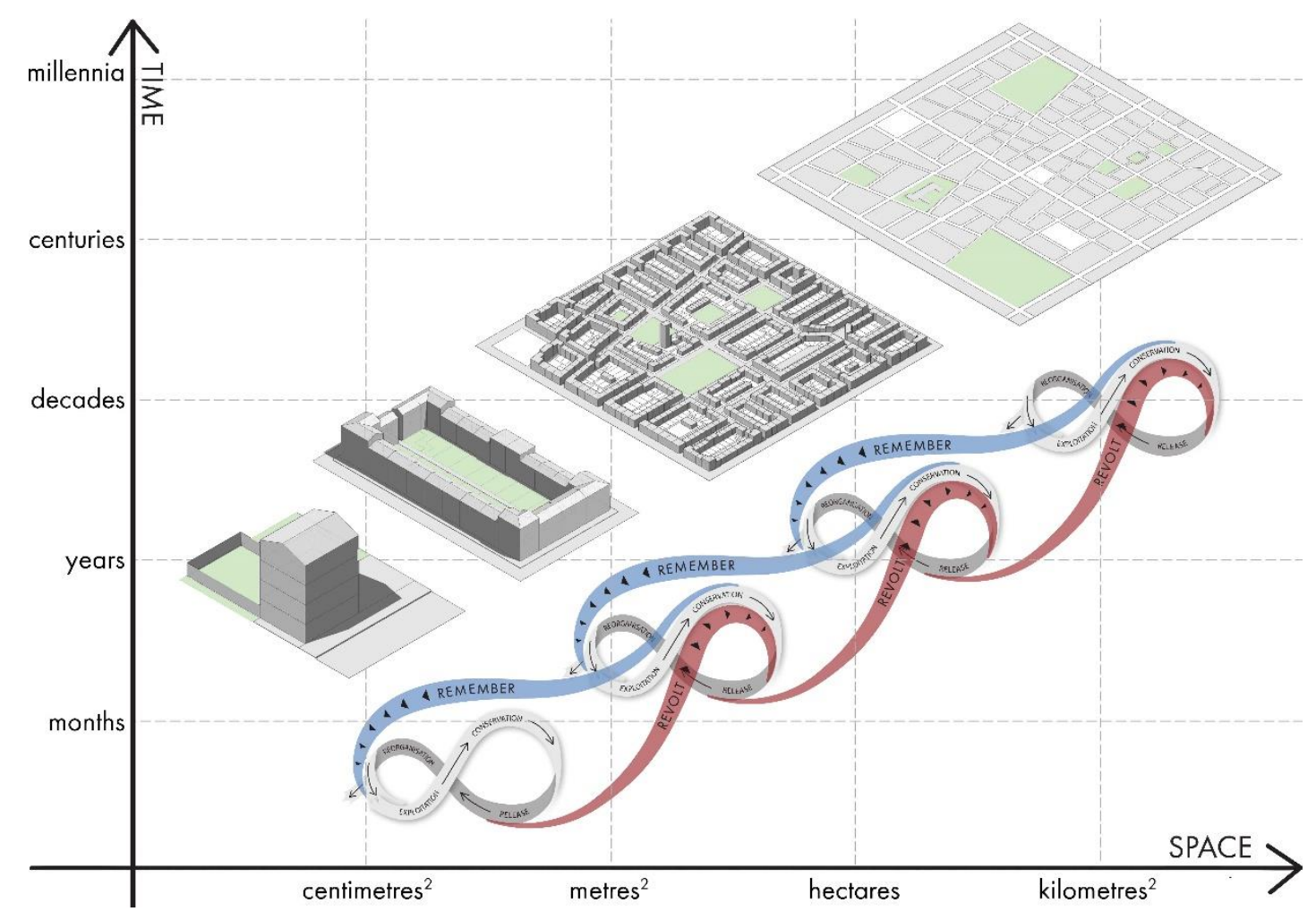

Feliciotti A., 2018, Resilience and Urban Design: A systems approach to the study of resilience in urban form. $\mathrm{PhD}$ Thesis, Department of Architecture, University of Strathclyde.

From this perspective, just like in ecosystems, it becomes clear that also in urban form there are slow, intermediate and fast variables: streets have a stabilizing effect, can last thousands of years and constrain the reconfiguration of smaller-scale morphological elements (Moudon, 1986); plots are more changeable but sufficiently stable to limit radical spatial change in the short time and their development cycle can take several hundred years; buildings, depending upon the durability of structure and materials, have an average lifespan comprised between 30-200 years and introduce frequent novelty in the urban fabric; building interiors and uses within them change as fast as their occupants, between months and decades. The complex spatial-temporal system of relationships between scales described earlier is the reason why we recognize a city footprint despite we might not recognize its individual buildings: we see a familiar order at a distance, but astounding variety up close (Figure 5).

Even in urban form, small-scale fast variables have limited capacity to trigger systemic change, but there are cases where bottom-up processes can trigger wide-ranging transformations, a notion that is common in practices of "urban acupuncture", which exploit the catalytic power of strategically-located small-scale spatial interventions. In this sense, just like in the Panarchy, conservative scales "remember" while dynamic scales "revolt". 
Figure 5. Satellite view of Eixample district, Barcelona and close-up on internal courtyards: despite progressive building refurbishment, extension and replacement, the overall structure of blocks and streets is virtually unchanged after over two Centuries, maintaining a high degree of coherence whilst still allowing a high variety of plot sizes and diversity of architectural styles.
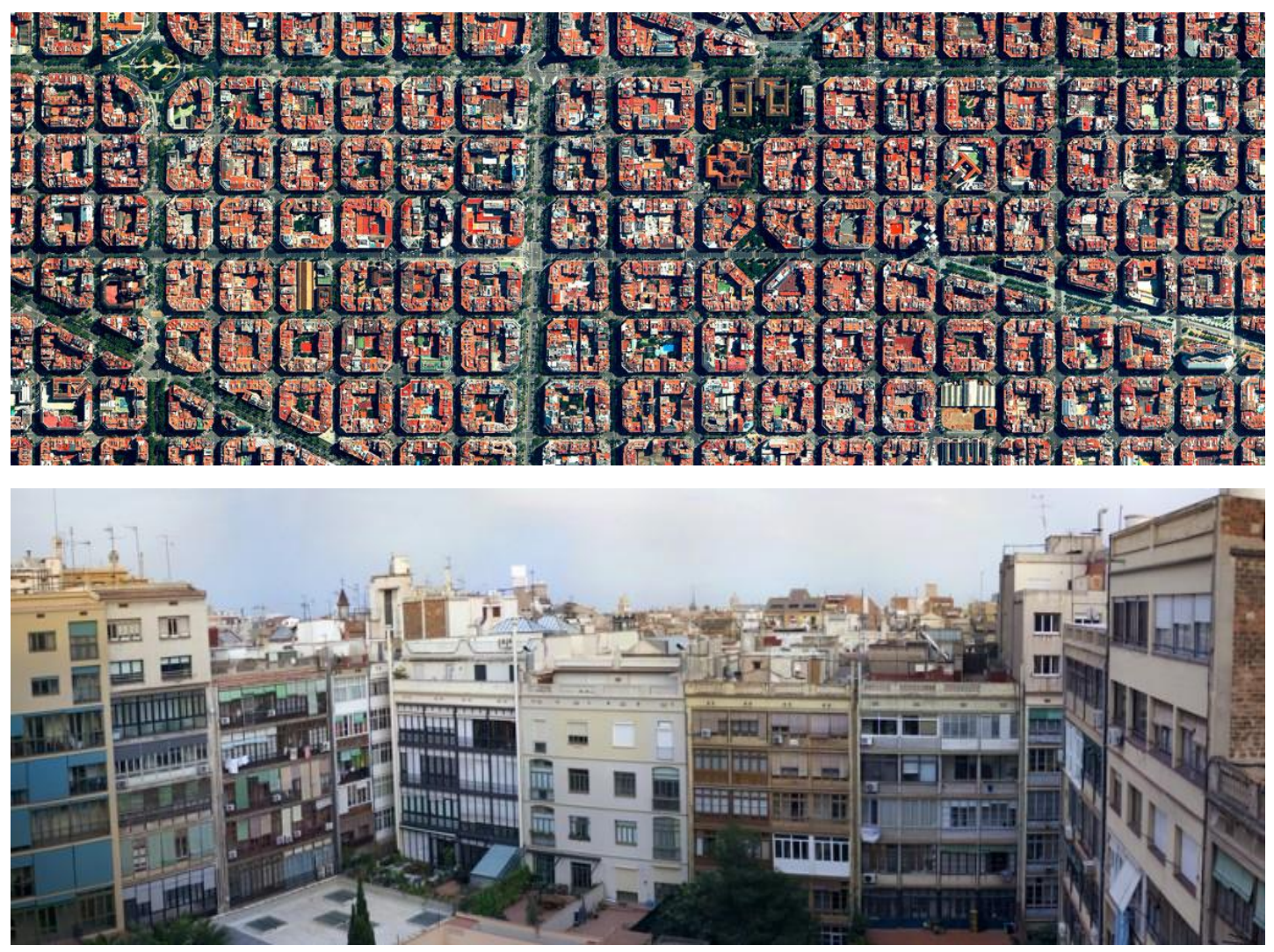

Source: Google Maps, (2017) and failedarchitecture.com.

\section{Getting to the point...}

It was Holling and Goldberg as early as 1971 that first suggested that urban systems and ecological systems share some common properties - including resilience - and that cities are prime examples of self-organising complex adaptive systems. Here, aided by Urban Morphology, we have shown that it is possible to extend this same view to urban form, as this appears to be structured and behave just like other kinds of complex adaptive systems. We have seen, that urban form is not a static phenomenon or a passive layer simply reacting to contextual factors, but is a dynamic entity subject to multiple cycles of change and strongly influenced by its historical development and past states, that has "its own weight and inertia, that work to oppose social, economic and political factors".

Crucially, this means that talking about resilience in the context of urban form is all but arbitrary. Quite on the contrary, we should carefully think about it whenever we are dealing with its design and management. The dualism between small and large, fast and slow, conservative and innovative inherent in the Panarchy, is at the very core of resilience, particularly in its evolutionary connotation: " $\underline{c o m p l e x}$ systems are resilient, because they appear to resist change or change slowly despite the interchange and 
evolution of individual components and the relationships between these components". This realization requires the adoption of a new awareness when designing and managing urban forms: it requires true time-consciousness. Whilst obviously urban form is just one aspect in the wider discourse on urban resilience, a deeper understanding of its specific contribution could bring important insights to address what Un-Habitat defined as one of the greatest challenges of our Century: the quest for new approaches to the design and management of sustainable and resilience cities.

\section{Conflict of Interest}

The authors declare no conflict of interest.

(C) 2018 by the authors; licensee MDPI and IFoU, This article is an open access article distributed under the terms and conditions of the Creative Commons Attribution license. 